\title{
Validation of prognostic scoring and assessment of clinical benefit for patients with bone sarcomas enrolled in phase I clinical trials
}

J. Andrew Livingston ${ }^{1}$, Kenneth R. Hess ${ }^{2}$, Aung Naing ${ }^{3}$, David S. Hong ${ }^{3}$, Shreyaskumar Patel ${ }^{4}$, Robert S. Benjamin ${ }^{4}$, Joseph A. Ludwig ${ }^{4}$, Anthony Conley ${ }^{4}$, Cynthia E. Herzog ${ }^{5}$, Pete Anderson ${ }^{6}$ Funda Meric-Bernstam ${ }^{3}$, Razelle Kurzrock ${ }^{7}$ and Vivek Subbiah ${ }^{3}$

\footnotetext{
1 Division of Cancer Medicine, The University of Texas MD Anderson Cancer Center, Houston, Texas, USA

2 Department of Biostatistics, The University of Texas MD Anderson Cancer Center, Houston, Texas, USA

${ }^{3}$ Department of Investigational Cancer Therapeutics (Phase I Clinical Trials Program), The University of Texas MD Anderson Cancer Center, Houston, Texas, USA

${ }^{4}$ Department of Sarcoma Medical Oncology, The University of Texas MD Anderson Cancer Center, Houston, Texas, USA

${ }^{5}$ Department of Pediatrics, The University of Texas MD Anderson Cancer Center, Houston, Texas, USA

${ }^{6}$ Pediatric Hematology Oncology, The Cleveland Clinic, Cleveland, Ohio, USA

${ }^{7}$ Experimental Therapeutics Program, University of California - San Diego, San Diego, California, USA
}

Correspondence to: Vivek Subbiah, email: vsubbiah@mdanderson.org

Keywords: bone sarcoma; phase 1 trials; prognosis scores; Ewing sarcoma; osteosarcoma

Received: June 13,2016 Accepted: June 26, $2016 \quad$ Published: July 28, 2016

\section{ABSTRACT}

Background: We sought to validate the Royal Marsden Hospital (RMH) and MD Anderson Cancer Center (MDACC) prognostic scoring systems for the selection of bone sarcoma patients for phase I clinical trials and to identify additional risk factors related to survival.

Patients and Methods: We retrospectively reviewed the baseline characteristics and outcomes of 92 bone sarcoma patients who were referred to MDACC's Phase I Clinical Trials Program.

Results: Ninety-two patients with Ewing sarcoma $(N=47)$, osteosarcoma ( $N=$ 22), chondrosarcoma $(N=16)$, and other tumors $(N=7)$ were evaluated; 78 were enrolled in at least 1 of $\mathbf{4 3}$ different phase I trials. The median overall survival (OS) was 8.8 months (95\% confidence interval $[\mathrm{CI}]=6.8-13.7$ months). Independent factors that predicted shorter survival were male sex, $>2$ metastatic sites, $>3$ previous therapies, hemoglobin level $<\mathbf{1 0 . 5} \mathrm{g} / \mathrm{dL}$, platelet count $>200 \times 10^{3} / \mathrm{L}$, creatinine level $\geq 1.3 \mathrm{mg} / \mathrm{dL}$, and lactate dehydrogenase level >ULN. Patients with good RMH scores $(0-1)$ had longer OS than patients with poor RMH scores $(2-3)(H R=5.8,95 \% \mathrm{CI}=$ 2.9-11.0; $P<0.0001)$, as did patients with low MDACC scores $(0-1)$ as compared to patients with higher MDACC scores $(2-4)(H R=3.2,95 \% C I=1.9-5.6 ; P<0.0001)$.

Conclusion: The RMH prognostic score can be used to predict the os of bone cancer patients referred for phase I trials. The MDACC score added no value to the RMH score and therefore does not have a role in assessment of patients with bone tumors. Patients with advanced bone sarcomas should be considered for phase I trials. 


\section{INTRODUCTION}

Bone sarcomas are rare tumors, accounting for $<$ $0.2 \%$ of all cancers. In the United States in 2014, about 3,000 patients were newly diagnosed with bone sarcomas, and more than 1,400 deaths were attributed to these tumors. [1] Patients with aggressive bone sarcomas, especially patients with advanced, metastatic, or relapsed disease, have an extremely poor prognosis $[2,3]$. With the exception of the immunotherapy mifamurtide, which has been approved in the European Union but is not available in the United States [4], no new systemic therapies have been approved for the treatment of the most prevalent bone sarcomas (osteosarcoma, Ewing sarcoma, and chondrosarcoma) in the last 3 decades.

In the United States, cytotoxic chemotherapy remains the standard of care for patients with osteosarcoma or Ewing sarcoma and altering these regimens or increasing their dose intensity has not improved overall survival (OS) for these groups in $>25$ years. [5] Similarly, patients with chondrosarcomas, which are resistant to the conventional chemotherapies that are effective against other bone sarcomas, have not seen improvement in their OS in $>30$ years. [6] Reasons for the lack of progress in developing effective therapies specifically against bone sarcomas include the rarity of these tumors as well as the complex and heterogeneous tumor biology which make the delivery of targeted therapy even more challenging [7, $8]$.

Enrolling patients with advanced, relapsed, or refractory bone cancers in phase I trials of targeted therapies may yield some unique insight into previously unexplored therapeutic targets, provide an opportunity to identify signals of early treatment response in these patients, and benefit these patients clinically $[9,10]$. However, many clinicians who screen such patients for early-phase clinical trials are often unable to accurately determine the survival profiles of individual patients, quite often an eligibility criterion for the study. Scoring systems that take prognostic factors into account have been established to optimize clinical decision-making regarding patients with advanced cancer. One of these systems, the Royal Marsden Hospital (RMH) scoring system, which is based on 3 survival-associated variables (albumin level, lactate dehydrogenase [LDH] level, and number of metastases), can be used to predict individual patient survival in phase I trials. [11] In a previous study, we validated the RMH scoring system for cancer patients treated at MD Anderson Cancer Center (MDACC). [12] The identification of additional independent prognostic factors for survival in that study led to a modification of the RMH scoring system and the development of the MDACC prognostic scoring system, which considers tumor type and ECOG performance status in addition to albumin level, LDH level, and number of metastases. [13] Both the RMH and MDACC prognostic scoring systems were developed and validated in patients with carcinomas and included few, if any patients with bone sarcomas. Thus, whether these systems can be used to predict outcomes in bone sarcoma patients is unknown. Validating either scoring system for bone sarcoma patients would provide clinicians with a valuable tool for selecting these patients for early-phase clinical trials.

Therefore, the objective of the present study was to validate these predictive models in bone sarcoma patients. We also sought to identify additional risk factors related to OS in this group and determine the clinical benefit these patients derive from participating in phase I trials to better inform physicians who refer bone sarcoma patients for early-phase clinical trial participation.

\section{MATERIALS AND METHODS}

\section{Patients}

We retrieved the electronic records of 92 consecutive patients who were referred to MDACC's Phase I Clinical Trials Program between July 2005 and November 2013 for evaluation for treatment in a phase I clinical trial. We reviewed the patients' records for the history, laboratory, and clinical findings at the time of presentation to the program, the treatment(s) given, and clinical outcomes. Pathology was reviewed by an MDACC pathologist with expertise in bone sarcomas in all cases. The therapies investigated in the clinical trials varied over time and were dependent on protocol availability at the time of presentation.

We recorded patients' baseline characteristics at referral to the program, including age, sex, tumor type, Eastern Cooperative Oncology Group (ECOG) performance status, number of prior systemic therapies for metastatic disease, number of sites of metastases, hemoglobin level $(\mathrm{g} / \mathrm{dL})$, LDH level $(\mathrm{U} / \mathrm{L})$, platelet count $(\mathrm{k} / \mathrm{uL})$, and albumin level $(\mathrm{g} / \mathrm{dL})$, as well as date of phase I therapy initiation, best response to phase I therapy based on Response Evaluation Criteria in Solid Tumors (RECIST; version 1.1), [14] and date of death or last follow-up. This study was approved by MDACC's Institutional Review Board (IRB). All clinical trials were also reviewed and approved by the institutional IRB. Patients provided their written informed consent to receive treatment on the respective phase I protocols.

\section{Statistical analysis}

Patient characteristics were summarized using medians and ranges for continuous variables and frequencies and percentages for categorical variables.

To test the RMH prognostic scoring system, we classified patients according to 3 variables: LDH level 
(normal [0] vs. $>$ upper limit of normal [ULN; +1]), albumin level $(\geq 3.5 \mathrm{~g} / \mathrm{dL}[0] v s .<3.5 \mathrm{~g} / \mathrm{dL}[+1])$, and number of metastatic sites of disease, $(\leq 2[0]$ vs. $>2[+1])$. The values for each variable were summed to provide a prognostic score, and patients were classified as having a good prognosis (RMH score of 0 or 1 ) or poor prognosis (RMH score of 2 or 3 ). To test the MDACC prognostic scoring system, we classified patients according to the $3 \mathrm{RMH}$ variables plus ECOG performance status (0 [0] $v s . \geq 1[+1]$ ). (Although the MDACC system includes gastrointestinal tumor type, this variable was not included in our analysis, as all patients had bone sarcomas.)

Progression-free survival (PFS) was measured from the time of clinical trial enrollment until imaging demonstrating disease progression by RECIST or last follow-up. OS was measured from the time of presentation to the phase I program until death from any cause or last follow-up. Median OS durations were estimated using the Kaplan-Meier method. [15] Patients were censored at the time of their last follow-up. Univariate Cox proportional hazard analysis was used to compare OS among subgroups of patients.

Univariate and multivariate Cox proportional hazards models were fit to assess associations among patient characteristics and clinical outcomes. [16] The covariates in the univariate analysis included age $(<40 v s$. $\geq 40$ years), sex, body surface area $\left(<2 v s . \geq 2 \mathrm{~m}^{2}\right)$, tumor type (Ewing sarcoma, osteosarcoma, chondrosarcoma, or other bone sarcoma), ECOG performance status $(0$,
1, 2, or 3), hemoglobin level ( $<10.5 v s . \geq 10.5 \mathrm{~g} / \mathrm{dL})$, platelet count ( $<200$ vs. $\left.\geq 200 \times 10^{3} / \mathrm{L}\right)$, albumin level ( $<3.5 v s . \geq 3.5 \mathrm{~g} / \mathrm{dL})$, creatinine level $(<1.3 v s . \geq 1.3 \mathrm{mg} /$ $\mathrm{dL})$, number of prior therapies $(<3 v s . \geq 3$ ), history of prior radiation (yes $v s$. no), number of metastases $(\leq 2 v s$. $>2)$, LDH level $(\leq 618 v s .>618 \mathrm{U} / \mathrm{L}), \mathrm{RMH}$ score, and MDACC score. These variables were measured at the time of presentation to the phase I program. Variables with $p>$ 0.50 were removed from the model due to sample size. Cox proportional hazards analysis was used to validate the $\mathrm{RMH}$ and MDACC prognostic scores using our data set. All statistical tests were 2 -sided, and $P$ values $<0.05$ were considered statistically significant. Statistical analyses were conducted with SAS statistical software (version 9.1; SAS Institute Inc., Cary, NC).

\section{RESULTS}

\section{Patient characteristics}

Ninety-two patients (58 men [63\%] and 34 women [37\%]) with bone sarcomas who were evaluated for participation in phase I clinical trials in MDACC's Phase I Clinical Trials Program were included in this retrospective review. These patients' baseline characteristics at referral to the program are given in Table 1. The most common tumor type was Ewing sarcoma in 47 patients

\section{PFS by Histology}

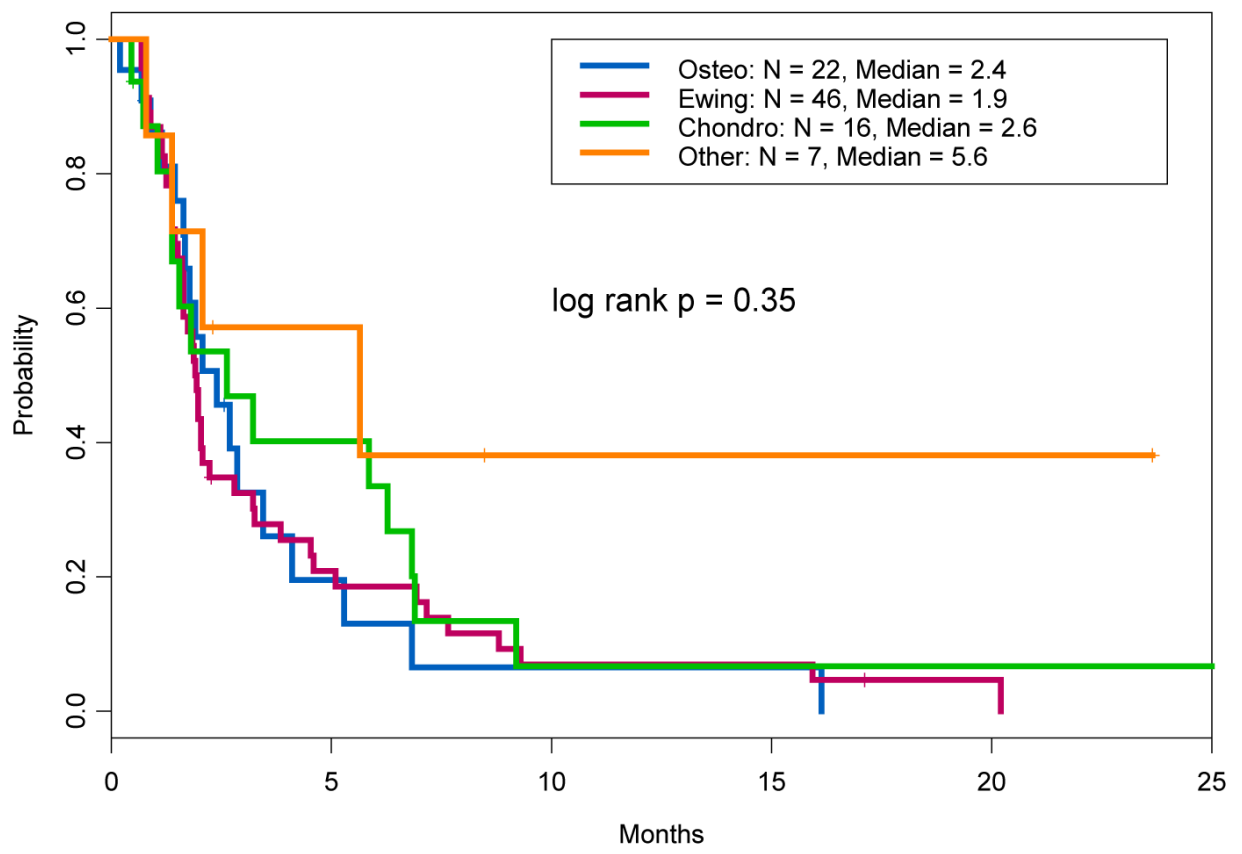

Figure 1: Progression-free survival by tumor type. 
Table 1: Baseline characteristics of the 92 bone sarcoma patients in the present study.

\begin{tabular}{|c|c|}
\hline Characteristic & No. of Patients (\%) \\
\hline \multicolumn{2}{|l|}{ Sex } \\
\hline Male & $58(63)$ \\
\hline Female & $34(37)$ \\
\hline \multicolumn{2}{|l|}{ Age, years } \\
\hline Median & 24 \\
\hline Range & $11-79$ \\
\hline$<18$ & $11(11)$ \\
\hline $18-39$ & $53(58)$ \\
\hline $40-65$ & $18(20)$ \\
\hline$>65$ & $10(11)$ \\
\hline \multicolumn{2}{|l|}{ Tumor type } \\
\hline Ewing sarcoma & $47(51)$ \\
\hline Osteosarcoma & $22(24)$ \\
\hline Chondrosarcoma & $16(17)$ \\
\hline Chordoma & $5(5)$ \\
\hline Other & $2(3)$ \\
\hline \multicolumn{2}{|c|}{ ECOG performance status } \\
\hline 0 & $25(27)$ \\
\hline 1 & $57(62)$ \\
\hline 2 & $7(8)$ \\
\hline \multicolumn{2}{|c|}{ No. of prior chemotherapies } \\
\hline Median & 3 \\
\hline Range & $0-11$ \\
\hline $0-2$ & $47(51)$ \\
\hline$\geq 3$ & $45(49)$ \\
\hline \multicolumn{2}{|c|}{ Prior radiation therapy } \\
\hline Yes & $57(62)$ \\
\hline No & $35(38)$ \\
\hline \multicolumn{2}{|c|}{$\begin{array}{l}\text { Prior immunotherapy, including } \\
\text { monoclonal antibodies }\end{array}$} \\
\hline Yes & $12(13)$ \\
\hline No & $80(87)$ \\
\hline \multicolumn{2}{|l|}{ No. of metastases } \\
\hline$\leq 2$ & $66(72)$ \\
\hline$>2$ & $26(28)$ \\
\hline \multicolumn{2}{|l|}{ RMH score } \\
\hline 0 or 1 & $74(81)$ \\
\hline 2 or 3 & $17(19)$ \\
\hline \multicolumn{2}{|l|}{ MDACC score } \\
\hline 0 or 1 & $49(53)$ \\
\hline $2-4$ & $40(45)$ \\
\hline
\end{tabular}

RMH: Royal Marsden Hospital prognostic score, MDACC: MD Anderson Cancer Center prognostic score. 
Table 2: Mechanisms of action of agents in phase I trials enrolling patients in the present study.

\begin{tabular}{|l|}
\hline Mechanism of action \\
\hline Anthracycline + proteasome inhibitor + nucleoside analog \\
\hline Anti-IL1 monoclonal antibody \\
\hline Anti-microtubule agent + nucleoside analog + VEGF monoclonal antibody \\
\hline Aurora kinase inhibitor \\
\hline Biguanide + mTOR inhibitor \\
\hline CDK inhibitor \\
\hline cMet inhibitor \\
\hline cMET/Alk inhibitor + mulitargeted TKI \\
\hline Death receptor ligands \\
\hline EGFR TKI + EGFR monoclonal antibody \\
\hline HDAC inhibitor + multitargeted TKI \\
\hline HDAC inhibitor + VEGF inhibitor + mTOR inhibitor \\
\hline HER2 TKI + biguanide \\
\hline HIF inhibitor \\
\hline IgG1/delta3 monoclonal antibody \\
\hline Immune modulator + mTOR inhibitor \\
\hline IGF-1R antibody \\
\hline IGF-1R antibody + mTOR inhibitor \\
\hline Microtubule stabilizing agent + vitamin K antagonist \\
\hline mTOR inhibitor \\
\hline mTOR inhibitor + EGFR monoclonal antibody \\
\hline mTOR inhibitor + HDAC inhibitor \\
\hline Multitargeted TKI \\
\hline PARP inhibitor \\
\hline PI3K inhibitor \\
\hline Spliceosome inhibitor \\
\hline TRAIL receptor 2 antibody \\
\hline VEGF inhibitor + HER2 TKI \\
\hline VEGF monoclonal antibody + mTOR inhibitor \\
\hline VEGF TKI + VEGF monoclonal antibody \\
\hline
\end{tabular}

VEGF: vascular endothelial growth factor, mTOR: mechanistic target of rapamycin, EGFR: epidermal growth factor receptor, TKI: tyrosine kinase inhibitor, HDAC: histone deacetylase, HIF: hypoxia-inducible factor, IGF-1R: insulin-like growth factor 1 receptor, PARP: poly-ADP ribose polymerase, PI3K: phosphoinositide 3-kinase, TRAIL: TNF-related apoptosis-inducing ligand.

(51\%), followed by osteosarcoma in 22 patients $(24 \%)$, chondrosarcoma in 16 patients (17\%), and other tumors in 7 patients (8\%) (chordoma 5, 1 each hemangiopericytoma and malignant chondroid syringoma). The median patient age at referral was 24 years (range, 11-79 years). The median number of prior chemotherapy regimens was 3 (range, $0-11$ regimens), and 57 patients $(62 \%)$ had prior radiation therapy. All patients had either progressed on standard therapy or demonstrated progression in the absence of therapy for tumor types that lack effective standard of care treatments such as chondrosarcoma.

\section{Phase I trials}

Of the 92 patients referred for evaluation, 78 participated in at least 1 phase I trial (range, 1-6 trials). Patients participated in 43 different phase I trials, and 10 patients (11\%) participated in $>1$ trial. Of the 78 patients, $56(72 \%)$ were enrolled in trials that included a tyrosine kinase inhibitor or small-molecule targeted therapy, 33 $(42 \%)$ were enrolled in trials that included a targeted monoclonal antibody, and $9(12 \%)$ were enrolled in trials that included both a targeted agent and a cytotoxic agent. Most of the trials in which these patients were enrolled included 1-5 bone sarcoma patients total, with the exceptions of trials that included anti-insulin-like growth 
Table 3: Multivariate Cox regression model.

\begin{tabular}{|c|c|c|c|}
\hline Cox Model & HR & $95 \% \mathrm{CI}$ & $P$ \\
\hline Ewings vs. Osteosarcoma & 1.2 & $0.6,2.7$ & 0.56 \\
\hline Chondrosarcoma vs. Osteosarcoma & 0.8 & $0.3,2.2$ & 0.7 \\
\hline Other tumor type vs. Osteosarcoma & 0.1 & $0.3,0.6$ & 0.0084 \\
\hline Sex: male vs. female & 2.2 & $1.1,4.4$ & 0.025 \\
\hline Body surface area: $\geq 2$ vs. $<2$ & 1.7 & $0.8,3.4$ & 0.16 \\
\hline Number of metastatic sites: $>2 v s . \leq 2$ & 2.6 & $1.4,4.8$ & 0.0023 \\
\hline Number of prior therapies: $>3 v s . \leq 3$ & 1.9 & $1.0,3.5$ & 0.042 \\
\hline Hemoglobin: $<10.5 v s . \geq 10.5 \mathrm{~g} / \mathrm{dL}$ & 4.4 & $2.1,9.2$ & $<0.0001$ \\
\hline Platelets: $<200 v s .>200 \times 10^{3} / \mathrm{L}$ & 2.1 & $1.2,3.9$ & 0.015 \\
\hline Lactate dehyrdogenas (LDH): $\leq 618$ [normal] $v s .>618$ U/L $[>$ ULN] & 2.2 & $1.1,4.2$ & 0.018 \\
\hline Creatinine: $<1.3 v s . \geq 1.3 \mathrm{mg} / \mathrm{dL}$ & 0.4 & $0.1,0.9$ & 0.024 \\
\hline
\end{tabular}

Note: Age, ECOG PS, Bilirubin, and Albumin were removed from the model due to $p>0.50$. Adding Prior Radiation to the above model gives $\mathrm{HR}=0.5(0.3,1.0)$ with $p=0.070$.

factor 1 receptor (IGF-1R) antibodies alone (20 patients) or in combination with mammalian target of rapamycin (mTOR) inhibition (11 patients). A representative list of the mechanisms of action of the agents investigated in the clinical trials is shown in Table 2.

\section{Clinical outcomes}

The median progression-free survival (PFS) for all patients was 2.0 months (95\% confidence interval $[\mathrm{CI}]=$ 1.8-2.9 months). The 4-month PFS rates were $26 \%(95 \%$ $\mathrm{CI}=12-58 \%)$ for osteosarcoma patients, $26 \%(95 \% \mathrm{CI}$ $=16-42 \%$ ) for Ewing sarcoma patients, $40 \%$ (95\% CI $=22-74 \%)$ for chondrosarcoma patients, and 57\% (95\% $\mathrm{CI}=30-100 \%$ ) for patients with other tumor types (Figure 1). The median OS duration of all patients was 8.8 months $(95 \% \mathrm{CI}=6.8-13.7$ months $)$; the median OS durations of patients with osteosarcoma, Ewing sarcoma, and chondrosarcoma were 6.1, 8.5, and 13.2 months, respectively.

No patients had a complete response. Five patients $(6 \%)$ had a partial response, and 24 patients $(31 \%)$ had stable disease. Disease stabilization $>6$ months or partial response was seen in 12 patients (15\%). Three Ewing sarcoma patients had responses to anti-IGF-1R therapy with or without mTOR inhibition. Two chondrosarcoma patients had responses to the death receptor ligand therapy Apo2L/TRAIL (recombinant human protein apoptosis ligand 2/TNF-related apoptosis-inducing ligand or dulanermin), with one chondrosarcoma patient remaining on therapy with sustained response for $>5$ years. No osteosarcoma patients had a RECIST response. One Ewing sarcoma patient and one chondrosarcoma patient had prolonged disease stabilization for $>20$ months and $>$ 13 months, respectively.

\section{Validation of the RMH and MDACC prognostic scoring systems}

Our univariate analysis identified variables associated with shorter OS. Given the modest sample size, variables that were marginally significant in the univariate analysis, including age, ECOG performance status, bilirubin level, and albumin level $(P>0.50$ for all), were excluded from the multivariate analysis. Variables included in the final model are shown in Table 3. Independent factors that predicted shorter OS in the multivariate Cox model were male sex (hazard ratio [HR] $=2.2, P=0.025),>2$ sites of metastases $(\mathrm{HR}=2.6, P=$ $0.0023),>3$ previous therapies $(\mathrm{HR}=1.6, P=0.042)$, hemoglobin level $<10.5 \mathrm{~g} / \mathrm{dL}(\mathrm{HR}=4.4, P<0.0001)$, platelet count $>200 \times 10^{3} / \mathrm{L}(\mathrm{HR}=2.1, P=0.015)$, and LDH level $>$ ULN $(\mathrm{HR}=2.2, P=0.018)$. Normal renal function (serum creatinine $<1.3 \mathrm{mg} / \mathrm{dL}$ ) was favorable (HR $=0.4, P=0.024)$. When included in the model, patients who had prior radiation therapy tended to have longer OS, but this association was not significant $(\mathrm{HR}=$ $0.5, P=0.07)$.

The median OS duration of bone sarcoma patients who had RMH prognosis scores of 0 or 1 (15 months) was significantly longer than that of patients who had RMH scores of 2 or 3 ( 4 months; $\mathrm{HR}=5.8,95 \% \mathrm{CI}=1.9-5.6 ; P$ $<0.0001$ ) (Figure 2A). Similarly, the median OS duration of patients with MDACC prognosis scores of 0 or 1 (15 months) was significantly longer than that of patients who had MDACC scores of $2-4$ (5 months $\mathrm{HR}=3.2,95 \% \mathrm{CI}=$ 1.9-5.6; $P<0.0001$ ) (Figure 2B).

\section{DISCUSSION}

Our study suggests that the RMH prognostic scoring system can be used help appropriately select bone sarcoma patients for phase I trial participation. 
The prognostic variables we identified for bone sarcoma patients are similar to those identified for carcinoma patients. [11] Our analysis revealed that $\mathrm{RMH}$ variables, including $>2$ metastases and LDH level $>$ ULN, were associated with shorter OS in the Cox model. However, albumin level $<3.5 \mathrm{~g} / \mathrm{dL}$ was not associated with OS $(P>0.50)$, likely owing to the small proportion of study patients who had low albumin levels (4.4\%). In the initial analysis of the RMH scoring system for patients treated in phase I trials at MDACC, other variables, including $\geq 3$ prior therapies and elevated platelets were also found to have prognostic significance; however, only $4 \%$ of the patients included in that study were sarcoma patients, and the number of bone sarcoma patients was not

\section{(A)}

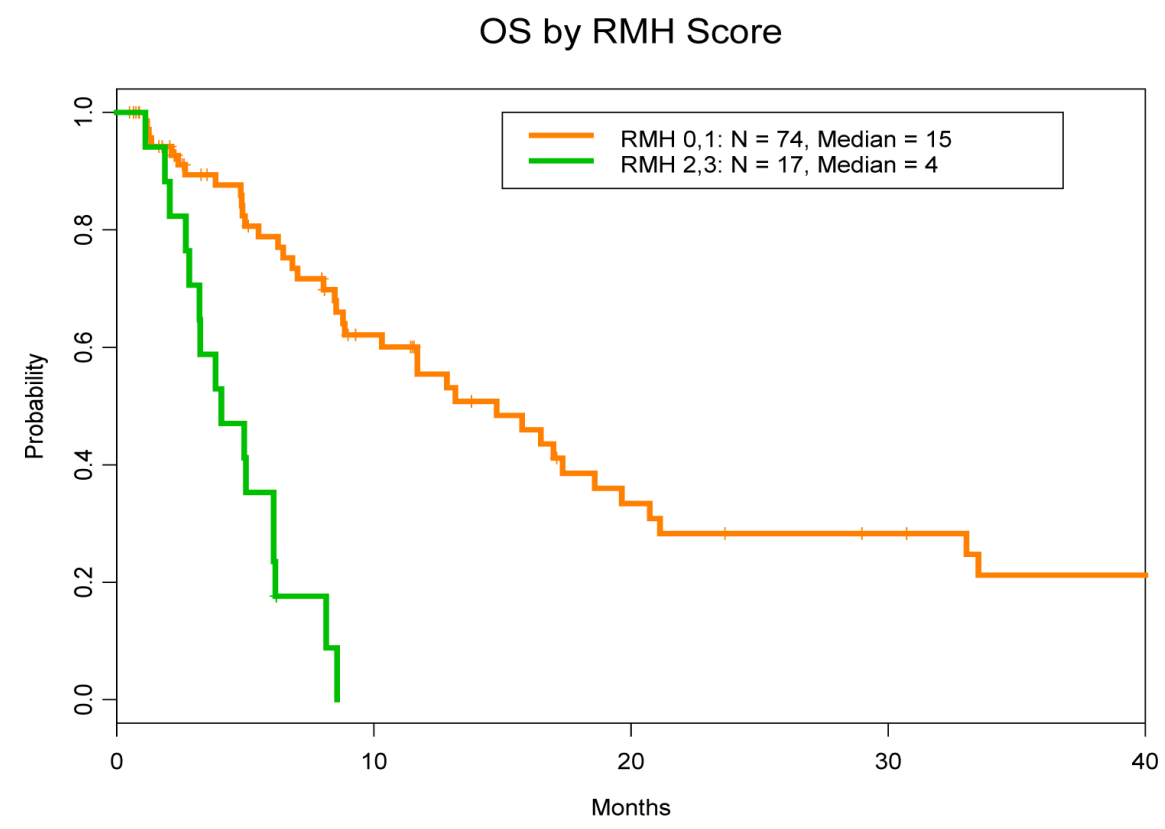

(B)

OS by MDACC Score

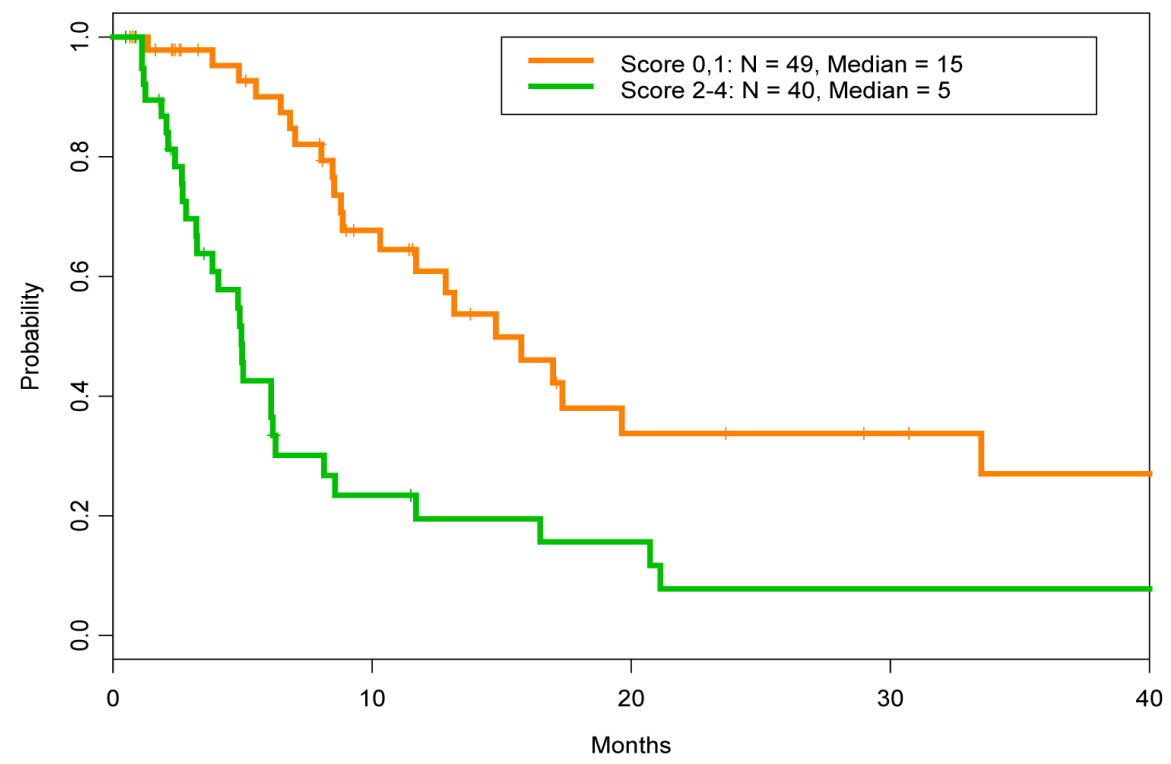

Figure 2: A. The median OS duration of bone sarcoma patients who had RMH prognosis scores of 0 or 1 (15 months) was significantly longer than that of patients who had RMH prognosis scores of 2 or 3 ( 4 months; $P<0.0001$ ). B. Similarly, the median OS duration of patients with MDACC prognosis scores of 0 or 1 (15 months) was significantly longer than that of patients who had MDACC prognosis scores of 2-4 (5 months; $P<0.0001)$. 
specified. In the present study, compared with the RMH scoring system, the MDACC system accounted for only 1 additional variable - ECOG performance status, which resulted in prognostic scores of 0-4, rather than 0-3-and thus did not add to the RMH score. In fact, it may have detracted, although not substantially. On the basis of these findings, we conclude that the RMH prognostic scoring system serves as an appropriate model to aid clinicians in the selection of bone sarcoma patients for referral to and participation in phase I clinical trials.

The present study's findings are similar to those of 2 other studies that evaluated the outcome, prognosis, and clinical benefit sarcoma patients derive from early-phase clinical trials. Jones et al. found that only elevated LDH levels and low albumin levels were significantly associated with OS duration. [17] The study included older patients (median age, 48 years) who predominantly had soft tissue sarcomas; $9 \%$ had chondrosarcomas and $<4 \%$ of patients had osteosarcomas. The study's authors acknowledged that they did not perform a subgroup analysis based on tumor histology, as the small number of patients in each group would have limited the usefulness of the findings. The RMH score was also validated in a pooled analysis of 178 sarcoma patients treated on phase I protocols. [18] This study also predominantly included patients with soft tissue sarcomas. It included only 22 patients (12.4\%) with aggressive bone sarcomas, and these patients were found to have the poorest outcomes, with a median OS duration of 16.6 weeks. Similarly, we found that the median OS durations of patients with Ewing sarcoma and osteosarcoma were significantly shorter than that of patients with chondrosarcoma, which is known to be a more indolent tumor.

Determining the clinical benefit of systemic therapies in phase I trials for bone sarcoma patients poses a unique challenge. First, because these trials are designed to evaluate safety rather than efficacy and second, many sarcomas-particularly osteosarcomas and chondrosarcomas - do not demonstrate dimensional responses [19]. This characteristic limits the utility of RECIST in identifying patients who are deriving a benefit from therapy. Often, alternative means of response assessment such as PET/CT imaging which can be useful in high grade bone sarcomas are not included in these trials. As with other tumor types, subtype specific phase II trials with appropriate endpoints are needed to confirm benefit. Some experts have suggested that PFS is a more appropriate measure of clinical benefit in sarcoma patients. $[20,21]$ Although benchmarks for a systemic therapy's activity against soft tissue sarcomas have been suggested, similar benchmarks for activity against advanced bone sarcomas have not been established. [22] With a lack of adequate response assessment for osteosarcoma or chondrosarcoma patients included in these studies, it is difficult to conclude whether the 4-month PFS reported in our study represents clinical benefit or simply reflects the natural history of these tumor types in the advanced setting. Our findings indicate that, despite the lack of responses in osteosarcoma patients, 4-month PFS may serve as a potential baseline for assessing the antitumor activity of future therapies against osteosarcomas. Responses to anti-IGF-1R therapy alone or in combination with mTOR inhibition in Ewing sarcoma patients have been reported, [23] as have responses to Apo2L/TRAIL therapy in chondrosarcoma patients. [24] When responses in Ewing sarcoma patients were seen, the availability of a promising agent increased referral of patients with bone sarcomas and rare tumors to early-phase clinical trials, which led to expansions of the phase I trials and subsequent phase II trials of anti-IGF-1R therapy alone [25-27] or in combination with mTOR inhibition. [28-30]

The major limitations of this study included its inclusion of patients with late-stage cancer, the referral bias inherent to phase I trials conducted in tertiary care centers, and the fact that patients received heterogeneous therapies. However, given the paucity of new drugs for patients with these rare diseases, any effort to enroll bone sarcoma patients in novel clinical trials to identify potential new drugs against the disease is worthwhile. During the time period of this study, additional Ewing sarcoma patients were treated on phase I trials of IGF1R therapies within the Pediatrics and Sarcoma Medical Oncology Departments within our institution; however these patients were not captured in this analysis. In addition, none of the patients in the present study were enrolled in trials of immune checkpoint inhibitors due to the time period of the study and the clinical trials available. There is significant enthusiasm for the potential efficacy of immune checkpoint inhibitors or other immunotherapies in bone sarcoma. Evaluation of these agents in the phase I and phase II setting is currently underway.

In summary, early-phase clinical trials are essential to developing therapies for patients with aggressivebiology bone sarcomas. Our findings, which validate the RMH prognostic scoring system in this group of patients, indicate that patients may derive a clinical benefit from participating in phase I trials, and that RMH prognostic scores in particular are useful in selecting bone sarcoma patients for such studies. Our findings reiterate the importance of considering bone sarcoma patients for phase I trials following the progression of their disease on standard therapy or, in the case of chondrosarcoma patients, at diagnosis if possible.

\section{ACKNOWLEDGMENTS}

Financial Support: The University of Texas MD Anderson Cancer Center is supported in part by a Cancer Center Support Grant (CA016672) from the National Institutes of Health. V.S. is supported by the Shanon Wilkes Osteosarcoma Research fund. 


\section{CONFLICTS OF INTEREST}

V.S. receives research support from Novartis, Bayer, GSK, Nanocarrier, Vegenics, Northwest Biotherapeutics, Berghealth, Incyte, Fujifilm, Pharmamar, D3, Pfizer, Amgen, and Abbvie.

\section{Editorial note}

This paper has been accepted based in part on peerreview conducted by another journal and the authors' response and revisions as well as expedited peer-review in Oncotarget

\section{Prior Presentation}

This research was presented in part at the Sarcoma Highlights Session at the 2014 American Society of Clinical Oncology Annual Meeting in Chicago, Illinois.

\section{REFERENCES}

1. Siegel R, Ma J, Zou Z and Jemal A. Cancer statistics, 2014. CA Cancer J Clin. 2014; 64:9-29.

2. Leary SE, Wozniak AW, Billups CA, Wu J, McPherson V, Neel MD, Rao BN and Daw NC. Survival of pediatric patients after relapsed osteosarcoma: the St. Jude Children's Research Hospital experience. Cancer. 2013; 119:26452653.

3. Stahl M, Ranft A, Paulussen M, Bölling T, Vieth V, Bielack S, Görtitz I, BraunMunzinger G, Hardes J and Jürgens H. Risk of recurrence and survival after relapse in patients with Ewing sarcoma. Pediatric blood \& cancer. 2011; 57:549553.

4. Meyers PA, Schwartz CL, Krailo MD, Healey JH, Bernstein ML, Betcher D, Ferguson WS, Gebhardt MC, Goorin AM and Harris M. Osteosarcoma: the addition of muramyl tripeptide to chemotherapy improves overall survival-a report from the Children's Oncology Group. Journal of Clinical Oncology. 2008; 26:633-638.

5. Smith MA, Seibel NL, Altekruse SF, Ries LA, Melbert DL, O'Leary M, Smith FO and Reaman GH. Outcomes for children and adolescents with cancer: challenges for the twenty-first century. Journal of Clinical Oncology. 2010; 28:2625-2634.

6. Giuffrida AY, Burgueno JE, Koniaris LG, Gutierrez JC, Duncan R and Scully SP. Chondrosarcoma in the United States (1973 to 2003): an analysis of 2890 cases from the SEER database. The Journal of Bone \& Joint Surgery. 2009; 91:1063-1072.

7. Egas-Bejar D, Anderson PM, Agarwal R, CorralesMedina F, Devarajan E, Huh WW, Brown RE and Subbiah V. Theranostic Profiling for Actionable Aberrations in
Advanced High Risk Osteosarcoma with Aggressive Biology Reveals High Molecular Diversity: The Human Fingerprint Hypothesis. Oncoscience. 2014; 1:167-179. doi: 10.18632/oncoscience. 21 .

8. Subbiah V, Wagner MJ, McGuire MF, Sarwari NM, Devarajan E, Lewis VO, Westin S, Kato S, Brown RE and Anderson P. Personalized comprehensive molecular profiling of high risk osteosarcoma: Implications and limitations for precision medicine. Oncotarget. 2015; 6:40642-40654. doi: 10.18632/oncotarget.5841.

9. Subbiah V. Prospects and pitfalls of personalizing therapies for sarcomas: from children, adolescents, and young adults to the elderly. Current oncology reports. 2014; 16:401.

10. Subbiah V and Kurzrock R. Phase 1 clinical trials for sarcomas: the cutting edge. Curr Opin Oncol. 2011; 23:352360 .

11. Arkenau H-T, Barriuso J, Olmos D, Ang JE, de Bono J, Judson I and Kaye S. Prospective validation of a prognostic score to improve patient selection for oncology phase I trials. Journal of Clinical Oncology. 2009; 27:2692-2696.

12. GarridoLaguna I, Janku F, Vaklavas C, Falchook GS, Fu S, Hong DS, Naing A, Tsimberidou AM, Wen S and Kurzrock R. Validation of the royal marsden hospital prognostic score in patients treated in the phase I clinical trials program at the MD Anderson Cancer Center. Cancer. 2012; 118:14221428.

13. Wheler J, Tsimberidou AM, Hong D, Naing A, Falchook G, Piha-Paul S, Fu S, Moulder S, Stephen B and Wen S. Survival of 1,181 patients in a phase I clinic: the MD Anderson Clinical Center for targeted therapy experience. Clinical Cancer Research. 2012; 18:2922-2929.

14. Eisenhauer E, Therasse P, Bogaerts J, Schwartz L, Sargent D, Ford R, Dancey J, Arbuck S, Gwyther S and Mooney M. New response evaluation criteria in solid tumours: revised RECIST guideline (version 1.1). European journal of cancer. 2009; 45:228-247.

15. Kaplan EL and Meier P. Nonparametric estimation from incomplete observations. Journal of the American statistical association. 1958; 53:457-481.

16. Cox D. R egression Models and Life Tables (with Discussion). JR Statist Soc B. 3:1.

17. Jones RL, Olmos D, Thway K, Fisher C, Tunariu N, PostelVinay S, Scurr M, de Bono J, Kaye SB and Judson IR. Clinical benefit of early phase clinical trial participation for advanced sarcoma patients. Cancer chemotherapy and pharmacology. 2011; 68:423-429.

18. Cassier P, Polivka V, Judson I, Soria J-C, Penel N, Marsoni S, Verweij J, Schellens J, Morales-Barrera R and Schöffski P. Outcome of patients with sarcoma and other mesenchymal tumours participating in phase I trials: a subset analysis of a European Phase I database. Annals of Oncology. 2014:mdu108.

19. Benjamin RS. SARC-CTOS imaging symposium: introduction to the problem from a clinical perspective. The 
oncologist. 2008; 13(Supplement 2):1-3.

20. Benjamin RS. Osteosarcoma: better treatment through better trial design. The Lancet Oncology. 2015; 16:12-13.

21. Grignani G, Palmerini E, Ferraresi V, D'Ambrosio L, Bertulli R, Asaftei SD, Tamburini A, Pignochino Y, Sangiolo D and Marchesi E. Sorafenib and everolimus for patients with unresectable high-grade osteosarcoma progressing after standard treatment: a non-randomised phase 2 clinical trial. The Lancet Oncology. 2015; 16:98107.

22. Van Glabbeke M, Verweij J, Judson I and Nielsen OS. Progression-free rate as the principal end-point for phase II trials in soft-tissue sarcomas. European Journal of Cancer. 2002; 38:543-549.

23. Subbiah V, Naing A, Brown RE, Chen H, Doyle L, LoRusso P, Benjamin R, Anderson P and Kurzrock R. Targeted morphoproteomic profiling of Ewing's sarcoma treated with insulin-like growth factor 1 receptor (IGF1R) inhibitors: response/resistance signatures. PLoS One. 2011; 6:e18424.

24. Subbiah V, Brown RE, Buryanek J, Trent J, Ashkenazi A, Herbst R and Kurzrock R. Targeting the apoptotic pathway in chondrosarcoma using recombinant human Apo2L/TRAIL (dulanermin), a dual proapoptotic receptor (DR4/DR5) agonist. Molecular cancer therapeutics. 2012; 11:2541-2546.

25. Pappo AS, Patel SR, Crowley J, Reinke DK, Kuenkele K-P, Chawla SP, Toner GC, Maki RG, Meyers PA and Chugh R. R1507, a monoclonal antibody to the insulin-like growth factor 1 receptor, in patients with recurrent or refractory Ewing sarcoma family of tumors: results of a phase II Sarcoma Alliance for Research through Collaboration study. Journal of Clinical Oncology. 2011; 29:4541-4547.
26. Tap WD, Demetri G, Barnette P, Desai J, Kavan P, Tozer R, Benedetto PW, Friberg G, Deng H and McCaffery I. Phase II study of Ganitumab, a fully human anti-type-1 insulin-like growth factor receptor antibody, in patients with metastatic ewing family tumors or desmoplastic small round cell tumors. Journal of Clinical Oncology. 2012; 30:1849-1856.

27. Malempati S, Weigel B, Ingle AM, Ahern CH, Carroll JM, Roberts CT, Reid JM, Schmechel S, Voss SD and Cho SY. Phase I/II trial and pharmacokinetic study of cixutumumab in pediatric patients with refractory solid tumors and Ewing sarcoma: a report from the Children's Oncology Group. Journal of Clinical Oncology. 2012; 30:256-262.

28. Naing A, LoRusso P, Fu S, Hong DS, Anderson P, Benjamin RS, Ludwig J, Chen HX, Doyle LA and Kurzrock R. Insulin growth factor-receptor (IGF-1R) antibody cixutumumab combined with the MTOR inhibitor temsirolimus in patients with refractory Ewing's sarcoma family tumors. Clinical Cancer Research. 2012; 18:26252631.

29. Quek R, Wang Q, Morgan JA, Shapiro GI, Butrynski JE, Ramaiya N, Huftalen T, Jederlinic N, Manola J and Wagner AJ. Combination mTOR and IGF-1R inhibition: phase I trial of everolimus and figitumumab in patients with advanced sarcomas and other solid tumors. Clinical Cancer Research. 2011; 17:871-879.

30. Schwartz GK, Tap WD, Qin L-X, Livingston MB, Undevia SD, Chmielowski B, Agulnik M, Schuetze SM, Reed DR and Okuno SH. Cixutumumab and temsirolimus for patients with bone and soft-tissue sarcoma: a multicentre, openlabel, phase 2 trial. The lancet oncology. 2013; 14:371-382. 